\title{
Altered Passive Eruption: Report on Management of Two Cases
}

\author{
${ }^{1}$ Seema Roodmal Yadav, ${ }^{2}$ Vishwas Madaan, ${ }^{3}$ Vineet Vaman Kini, ${ }^{4}$ Ashvini M Padhye
}

\begin{abstract}
Excessive gingival display while smiling is one of the significant esthetic concern for many patients. One of the etiology for this is altered passive eruption (APE). A clinician must have a thorough knowledge of the clinical presentation of this condition and methods to eliminate it in order to provide the desired esthetical and functional outcome. Periodontic plastic procedures provide the best solution to this condition not only by eliminating the excess of gingiva but also by enhancing the gingival esthetics and biological harmony.

This case report highlights the clinical presentation of alter passive eruption in two patients along with sequential approach for management of such condition.
\end{abstract}

Keywords: Altered passive eruption, Esthetics, Gingival.

How to cite this article: Yadav SR, Madaan V, Kini VV, Padhye AM. Altered Passive Eruption: Report on Management of Two Cases. J Contemp Dent 2015;5(3):173-177.

Source of support: Nil

Conflict of interest: None

\section{INTRODUCTION}

Periodontal plastic procedures play a very important role in achieving esthetic gingival harmony. The esthetic appearance of smile significantly depends on structure of lip form, Gingival elements like the gingival zenith and gingival display; and Smile symmetry. ${ }^{1}$ Magne and Belser ${ }^{2}$ highlighted relative tooth dimensions and color, tooth axis, smile symmetry, and incisal edge configuration as the important gingival and dental objectives which must be achieved while restoring the esthetics of maxillary anterior dentition. Garber and Salama ${ }^{3}$ emphasized the relationship between teeth and gingival tissue as a major component of the esthetic smile with gingival zeniths and balance of the gingival levels being the key factors for an esthetic smile. The gingival zenith lies at the same height for central incisors and canines,

\footnotetext{
${ }^{1,2}$ Postgraduate Student, ${ }^{3}$ Professor and Guide

${ }^{4}$ Professor, Guide and Head

${ }^{1-4}$ Department of Periodontics, MGM Dental College and Hospital, Navi Mumbai, Maharashtra, India
}

Corresponding Author: Seema Roodmal Yadav, Postgraduate Student, Department of Periodontics, MGM Dental College and Hospital, Navi Mumbai, Maharashtra, India, Phone: 9960850689, e-mail: dr.seema345@gmail.com whereas for lateral incisors it is slightly coronal to the central incisor. Allen suggested that 2 to $3 \mathrm{~mm}$ of gingival exposure was perceived as esthetically pleasing smile by patients, whereas an excessive exposure $(>3 \mathrm{~mm})$ was perceived as unesthetic. ${ }^{4}$ The various conditions in which gummy smile can be encountered include case of vertical maxillary excess; hypotonic short upper lip, hyperfunctional upper lip and combination of both. Other cause of excessive gingival display is insufficient clinical crown height. Insufficient crown height can be a result of traumatic injury, caries, attrition or coronally situated gingival complex either due to tissue hypertrophy or altered passive eruption (APE). Various endocrine dysfunctions, such as leading deficiency of growth hormone, insulin-like growth factors I and II, epidermal growth factor, hypopituitarism causing delayed dental eruption and are attributed as etiological factors for APE. ${ }^{5}$

The concept of delayed passive eruption was first introduced by Coslet et al in $1977^{6}$ describing the situation when the gingival margin does not migrate to its final position on the cemental surface, instead remains positioned on or near the enamel surface. Goldman and Cohen ${ }^{7}$ described it as the failure of the gingival tissue to adequately recede to a level apical to the cervical convexity of the crown.

Altered passive eruption is used synonymously with retarded passive eruption, incomplete passive eruption, delayed passive eruption, ${ }^{6,8}$ with a female predominance $(2: 1)^{9}$ and affecting persons in the age of 20 and 30 years. ${ }^{10}$

Altered passive eruption was classified by Coslet et $\mathrm{al}^{6}$ based on the relationship between the gingiva and the clinical crown on one hand and the relationship between the cementoenamel junction (CEJ) and the bone crest on the other. Types I and II are based on the gingival/ anatomic crown relationship.

Type I: The gingival margin is incisal to the CEJ, the dimension of keratinized gingiva is wider than usual, and clinical crowns are short.

Type II: The dimension of the gingiva from the gingival margin to the mucogingival junction appears normal. The free gingival margin is incisal or occlusal to the CEJ and the mucogingival junction is positioned at the CEJ.

Further these two types can have two subtypes depending upon the location of CEJ and alveolar crest. 
Subtype A: The distance of alveolar crest to CEJ is approximately $1.5 \mathrm{~mm}$ : in such cases a normal attachment can be found.

Subtype B: The alveolar crest is at the level of the CEJ or above.

This case report highlights the periodontal procedures for treating cases diagnosed with APE.

\section{CASE REPORTS}

\section{Case 1}

A 24 years old female patient reported with a chief complaint of excessive gingival display while smiling. There was no contributing medical or family history. Extraoral examination revealed no gross asymmetry, Leibert's class I denoting very high smile line (Fig. 1). ${ }^{11}$ Intraoral examination revealed no clinical signs of plaque induced periodontal disease.

Clinical examination revealed adequate attached gingiva in the maxillary anterior region, presence of pseudopockets along with gingival margin asymmetry between the two central incisors leading to short clinical crown height. Radiographic examination revealed $2 \mathrm{~mm}$ distance between the CEJ and alveolar bone. Based on these findings a diagnosis of type I subgroup A APE was made.

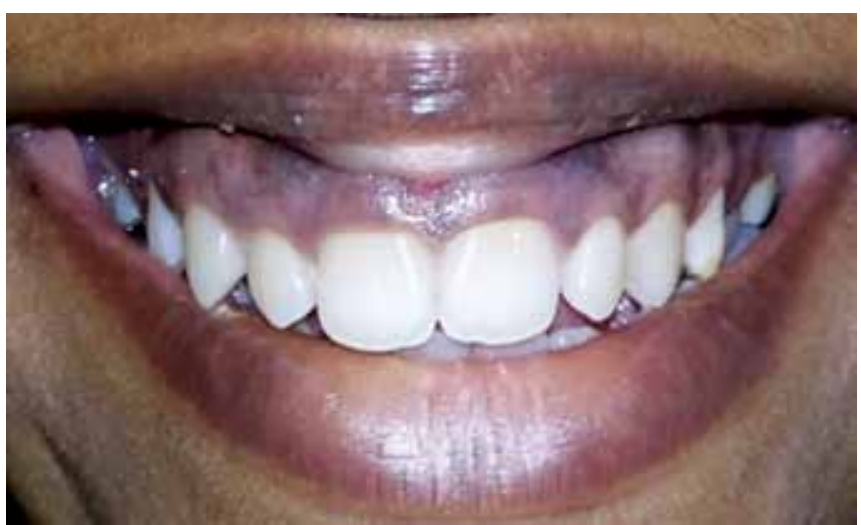

Fig. 1: Leiberts class 1: very high smile line with excessive gingival display preoperatively (case 1)

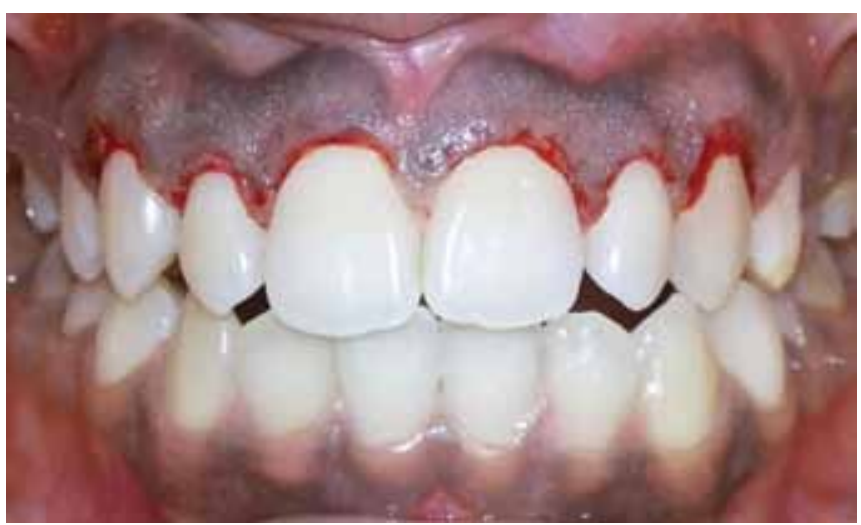

Fig. 3: External bevel gingivectomy performed (case 1)
Following the phase I periodontal therapy a decision to perform external bevel gingivectomy was made to improve the patient's esthetics. After obtaining the normal prerequisite values of blood investigations for periodontal surgery, an external bevel gingivectomy was undertaken in the region of 13 to 23.

Following the aseptic precautions under adequate local anesthesia a full-thickness continuous external bevel incision was placed using a scalpel to recreate the normal festooned pattern of gingiva, accompanied by removal of tissue from the facial surface, to complete the gingivectomy procedure; which also resulted in elimination of the pseudogingival pocket and increase clinical crown height (Figs 2 and 3). Gingival depigmentation was also carried out using scalpel so as to eliminate the gingival hyperpigmentation in order to further enhance the patients smile esthetics.

The postoperative period was uneventful. At the follow-up appointment after 1 week no postoperative sensitivity or pain were reported by the patient, and the patient was satisfied with the improved smile esthetics achieved (Figs 4 and 5).

\section{Case 2}

A 27 years old female patient reported with a chief complaint of short looking teeth and persistent redness

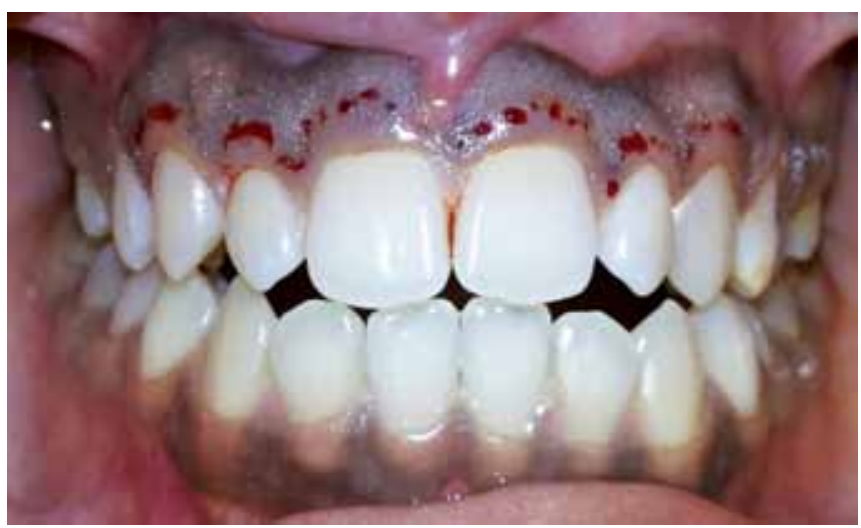

Fig. 2: Pseudo pockets marked (case 1)

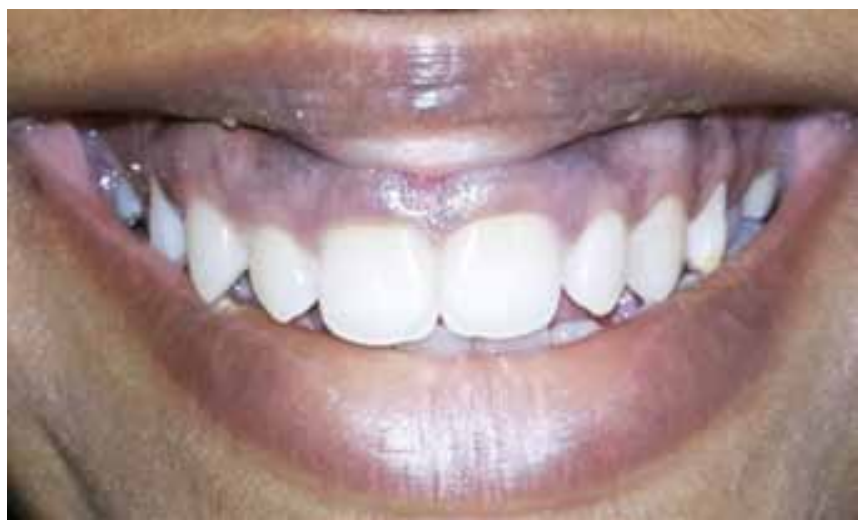

Fig. 4: Preoperative (case 1) 
of gums since past 5 to 6 months. Clinical examination revealed thick gingival morphotype with thick bulbous contour of gingiva and grade I gingival enlargement. The attached gingiva was found to be inadequate. Clinical probing highlighted the short clinical crown height of incisors (Fig. 6). Mucogingival junction was found to be located at 3 to $4 \mathrm{~mm}$ from the free gingival margin. Bone sounding revealed less than $2 \mathrm{~mm}$ distance between the crestal bone and CEJ. Radiographic examination revealed the presence of alveolar bone and CEJ at the same level (Fig. 7). A diagnosis of type II subgroup B APE was made considering the above the findings.

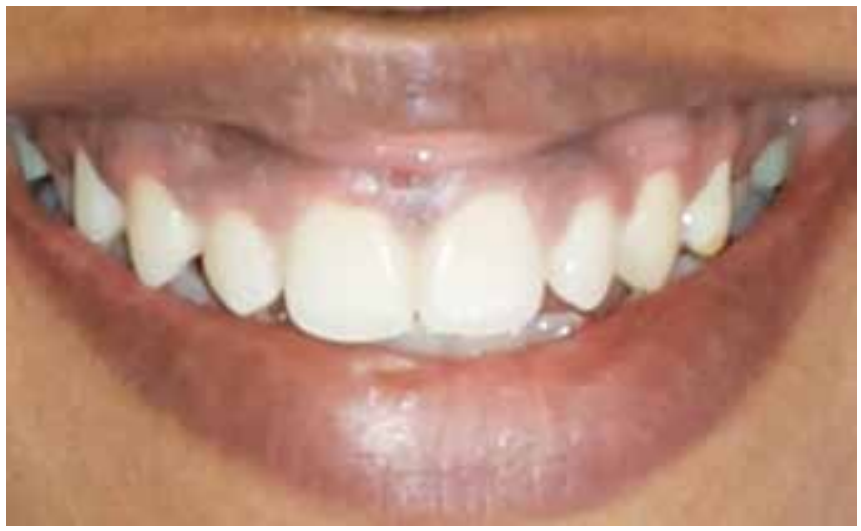

Fig. 5: Three months postoperative (case 2)
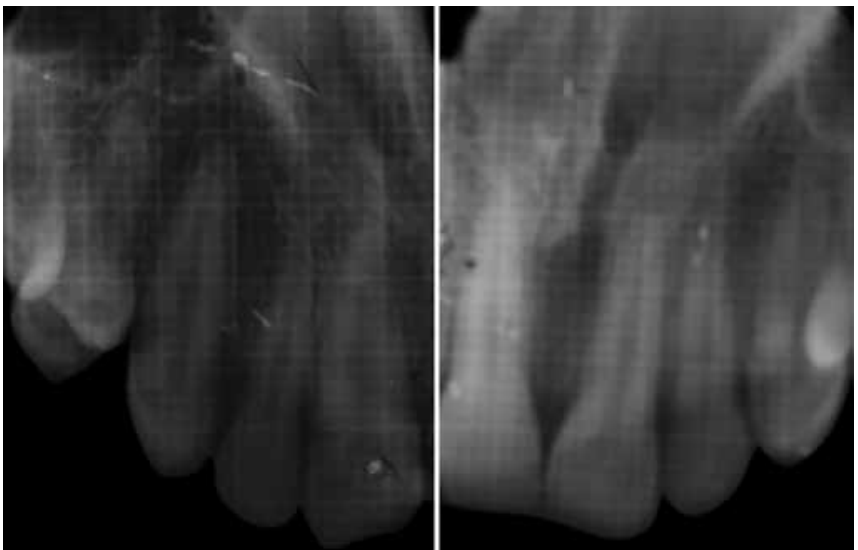

Fig. 7: Preoperative IOPA (case 2)

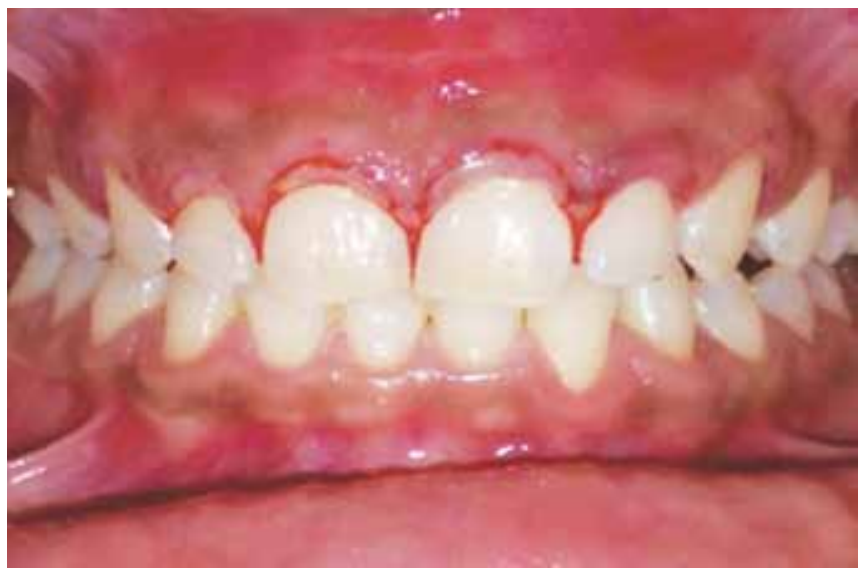

Fig. 9: Incisions given (case 2)
Following a successful phase I periodontal therapy a surgical intervention to increase the width of attached gingiva and to improve the gingival contours by apically displaced flap with osseous reduction was planned. Surgical phase was carried out using internal bevel incisions not involving the interdental papilla in order to obtain a uniform thickness of the flap and to allow for contouring of the buccal gingival margins in the maxillary anterior region. This was followed by placement of crevicular incisions at buttjoint to the interproximal papilla followed by two vertical releasing incisions extending beyond the mucogingival junction. A full thickness

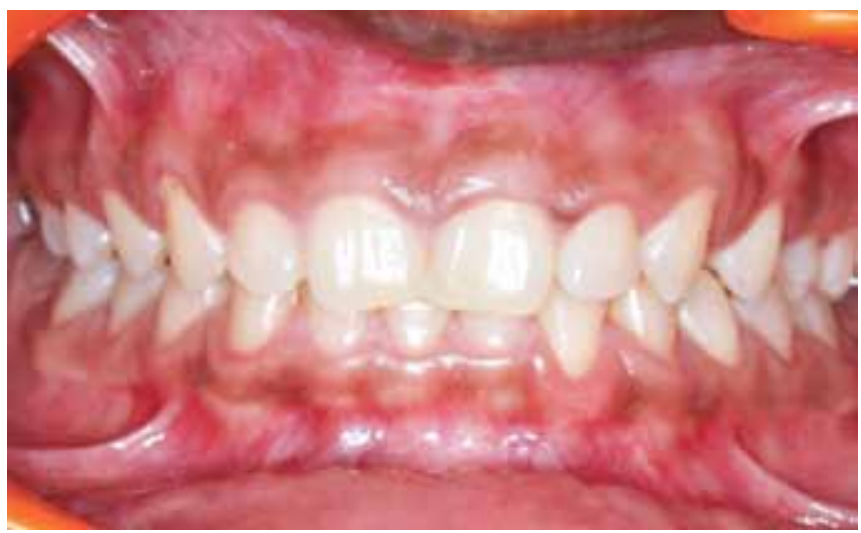

Fig. 6: Preoperative (case 2)

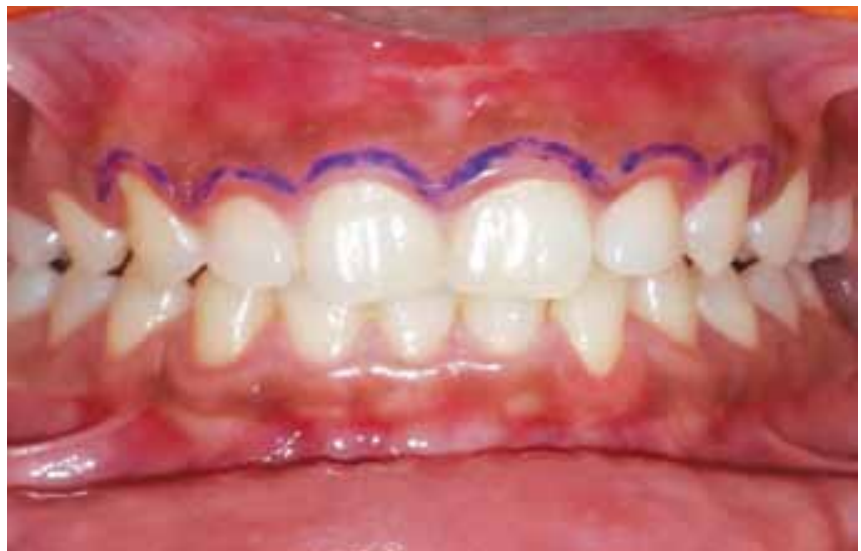

Fig. 8: Incision lines marked (case 2)

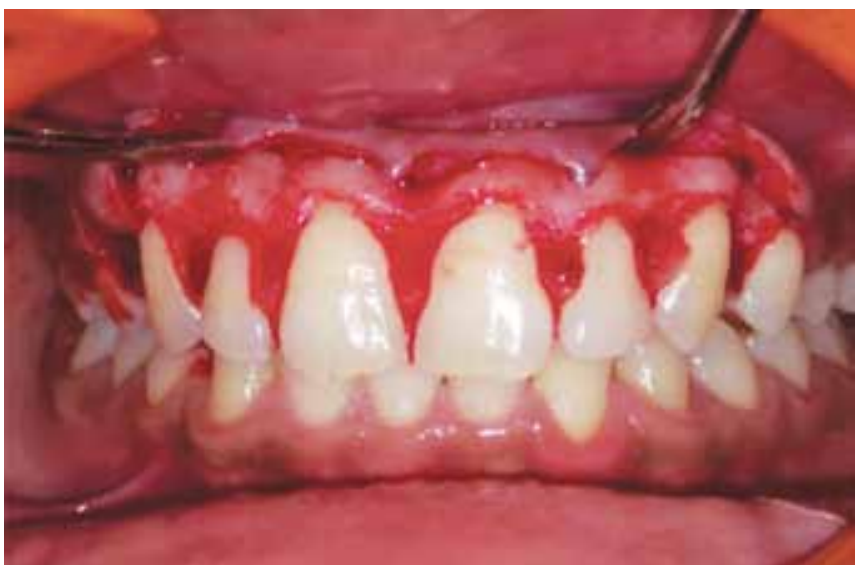

Fig. 10: Full thickness mucoperiosteal flap raised (case 2) 
mucoperiosteal flap was reflected (Figs 8 and 9) and the position of the CEJ was verified. Ostectomy was performed using Rotary and hand ostectomy instruments so as to establish the new biologic width by creating a distance of 2 to $2.5 \mathrm{~mm}$ between the crestal bone and CEJ. The bone was recontoured to reflect the soft tissue architecture. The flap was then apically repositioned to the new position and sutured (Figs 10 and 11). The postoperative period was uneventful. At the follow-up appointment after 1 week no postoperative sensitivity or pain were reported by the patient (Fig. 12). Patient was re-evaluated after 3 months which showed presence of pink health gingiva with knife edge margins with adequate attached gingiva along with the elimination of excessive gingival display.

\section{DISCUSSION}

The esthetic appearance of a smile is determined considering three main components: the teeth, the lip framework, and the gingival scaffold. Gingival shape and gingival contour are the two important aspects of gingiva that affect the final esthetic outcome. Gingival shape is the

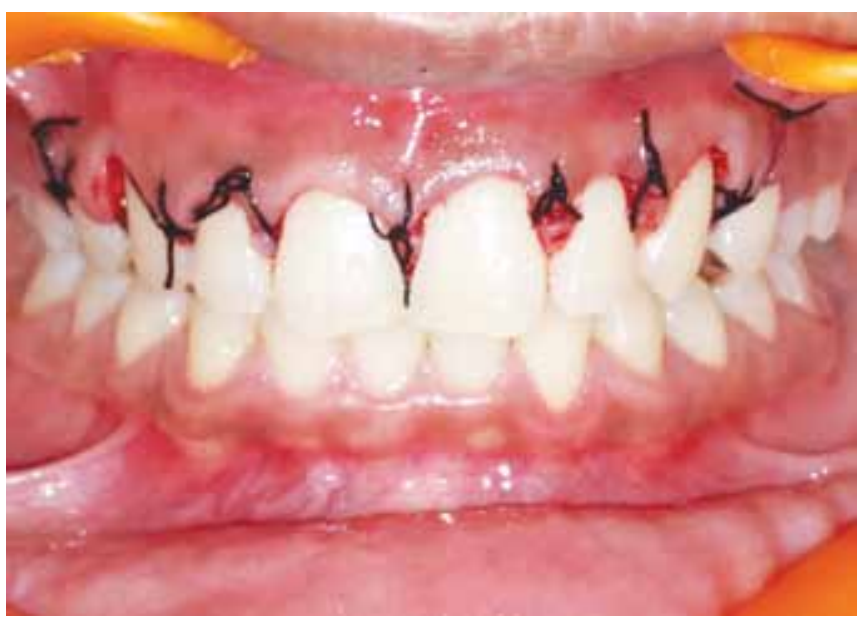

Fig. 11: Sutures placed (case 2)

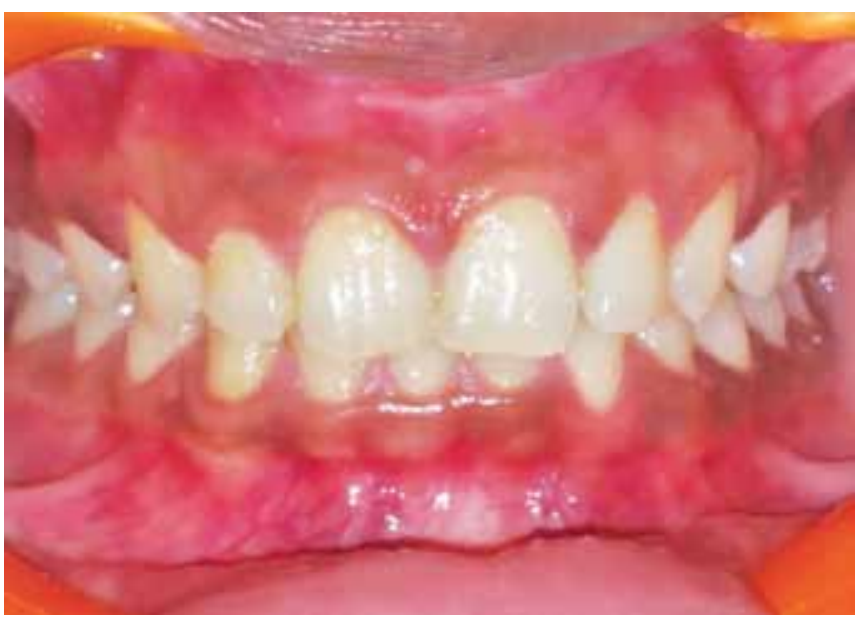

Fig. 12: Three months postoperative (case 2) curvature of the gingival margin of the tooth, determined by the CEJ and the osseous crest. ${ }^{12}$

One of the important aspect to treat this condition is to first diagnose it correctly and then classify it. Clinically, APE can be described as a condition where the free gingival margin is located greater than $2 \mathrm{~mm}$ coronal to the CEJ. Diagnosis begins with analyzing the gingival display while observing the patient in both smiling and reposing. While doing so the lip line of the patient whether high, medium, or low should also be noted as it influences the gingival display while smiling. ${ }^{11}$ To evaluate further the length and activity maxillary lip is recorded. The average length of the maxillary lip in repose is 20 to $22 \mathrm{~mm}$ in females and 22 to $24 \mathrm{~mm}$ in males. ${ }^{12,13}$ If the cause of a gummy smile is maxillary lip, there is no treatment necessary.

Further, clinical examination involves detection of CEJ. A explorer can be used to detect the CEJ. Location of the CEJ in the normal position in the gingival sulcus, suggests that the short clinical crown is probably the result of incisal wear. However, when the CEJ is not detected in the sulcus a diagnosis of APE can be made. This is followed by bone sounding which involves recording the distance from the free gingival margin to the alveolar crest which is approximately $3 \mathrm{~mm}$ in healthy unaltered gingiva. This distance governs whether ostectomy is necessary or not. ${ }^{14}$

Radiographic examination further facilitates the diagnosis of APE. If the radiograph reveals the clinical crown height to be less than the anatomical crown height, then the possibility of presence of APE should be considered (Flow Chart 1). ${ }^{15}$

A holistic approach for the treatment of APE involves considering the periodontal involvements, restorative and orthodontic requirements. ${ }^{16}$ When the clinical examination is suggestive of greater than $3 \mathrm{~mm}$ of tissue from bone to gingival crest, an adequate zone of attached gingiva a gingivectomy is sufficient to expose the hidden crown beneath the gingiva. An apically positioned flap with ostectomy is considered when the osseous levels are approximating the CEJ. Osseous recontouring also

Flow Chart 1: Clinical and radiographic evaluation of APE

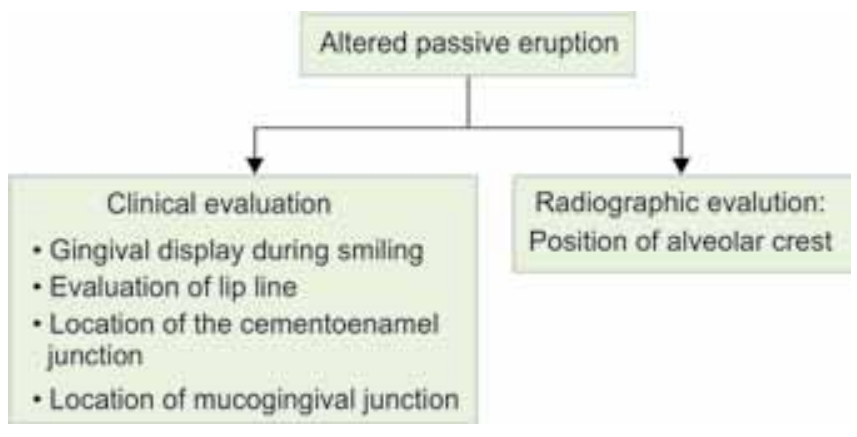


becomes necessary in cases where insufficient root is exposed to altering the biologic width. However, timing of periodontal surgery is a source for debate. Foley suggested orthodontic treatment to typically precede before the periodontal surgery, since the movement of teeth may affect the gingival harmony. ${ }^{17}$

Altered passive eruption is a genuine risk to periodontal health, and points to difficulties in oral hygiene and a narrow connective tissue attachment as possible causes. ${ }^{5}$ Volchansky and Cleaton-Jones ${ }^{9}$ reported a significant relationship between the presence of APE and acute necrotizing ulcerative gingivitis, suggesting that a the presence of deep gingival sulcus creates the necessary anaerobic conditions for the development of this infection. Altered passive eruption was also regarded as a clear risk situation for the periodontium in teeth that are going to be restored with total crowns or class II or III restorations by Dello Russo. ${ }^{18}$ The presence of a short clinical crown forces the clinician to make intrasulcular margin restorations; the difficulty of hygiene in this zone; and the absence of connective attachment to the radicular cement that can pose problems for the periodontal defenses.

\section{CONCLUSION}

Periodontal plastic surgery and osseous respective surgery lead to predictable outcomes in the treatment of APE. A careful preoperative planning enhances better surgical outcomes and enhances postsurgical stability of the gingival margin along with meeting the esthetic desire of the patients.

\section{REFERENCES}

1. Baiju CS, Khashu H, Garg A. Smile Design-periodontal outlook of basics. J Oral Health Comm Dent 2010;4:1-3.

2. Magne $P$, Belser U. Bonded porcelain restorations in the anterior dentition-a biomimetic approach. Quintessence Publishing Co, Chicago; 2002. p. 58-64.
3. Garber DA, Salama MA. The aesthetic smile: diagnosis and treatment. Periodontol 1996;11:18-28.

4. Allen EP. Use of mucogingival surgical procedure to enhance esthetics. Dent Clin North Am 1988;32:307-330.

5. Alpiste-Illueca F. Altered passive eruption (APE): a littleknown clinical situation. Med Oral Pathol Oral Cir Bucal 2011; 16(1):100-104.

6. Coslet JG, Vanarsdall R, Weisgold A. Diagnosis and classification of delayed passive eruption of the dentogingival junction in the adult. Alpha Omegan 1977;70(3):24-28.

7. Goldman HM, Cohen DW. Periodontal therapy, de 4 St Louis, CV Mosby Company; 1968.

8. Dolt AH 3rd, Robbins JW. Altered passive eruption: anetiology of short clinical crowns. Quintessence International 1977;28(6):363-372.

9. Volchansky A, Cleaton-Jones P. Delayed passive eruption-a predisposing factor for vincent's infection. J Dent Assoc S Afr 1974;29:291-294.

10. Silberberg N, Goldstein M, Smidt A. Excessive gingival display-Etiology, diagnosis and treatment modalities. Quintessence Int 2009;40:809-818.

11. Liébert MF, Fouque-Deruelle C, Santini A, Dillier FL, MonnetCorti V, Glise JM, Borghetti A. Smile line and periodontium visibility. Perio 2004;1:17-25.

12. Sarver DM. Principles of cosmetic dentistry in orthodontics: Part 1. Shape and proportionality of anterior teeth. Am J Orthodont Dentofac Orthoped 2004;126(6):749-753.

13. Peck S, Peck L. Esthetics and the treatment of the facial form. Ann Arbor, MI: University of Michigan; 1992. p. 97.

14. Moshrefi A. Altered passive eruption. J West Soc Periodontol Periodontal. Abstr 2000;48(1):5-8.

15. Hempton TJ, Esrason F. Crown lengthening to facilitate restorative treatment in the presence of incomplete passive eruption. J Calif Dent Assoc 2000;28(4):290-294.

16. Evian CI, Cutler SA, Rosenberg ES, Shah RK. Altered passive eruption: the undiagnosed entity. J Am Dent Assoc 1993;124(10):107-110.

17. Foley TF, Sandhu HS. Athanasopoulos C. Esthetic periodontal considerations in orthodontic treatment-the management of excessive gingival display. J Can Dent Assoc 2003;69(6): 368-372.

18. Dello Russo NM. Placement of crown margins in patients with altered passive eruption. Int J Periodont Rest Dent 1984; 4:58-65. 